\title{
Shape Changing Fabric Samples for Interactive Fashion Design
}

\author{
Daniela Ghanbari Vahid \\ Carleton University \\ Ottawa, Ontario, Canada \\ danivahid.dv@gmail.com \\ Audrey Girouard \\ Carleton University \\ Ottawa, Ontario, Canada \\ Audrey.Girouard@Carleton.ca
}

\author{
Lee Jones \\ Carleton University \\ Ottawa, Ontario, Canada \\ Lee.Jones@Carleton.ca \\ Lois Frankel \\ Carleton University \\ Ottawa, Ontario, Canada \\ Lois.Frankel@Carleton.ca
}

\begin{abstract}
As technology is integrated into all aspects of our lives, researchers are exploring emerging technologies in the field of fashion design. The substantial growth in the field of functional apparel design, such as the use of smart textiles, encourages researchers and fashion designers to incorporate technology within their designs. In much previous work, e-textiles have required interdisciplinary knowledge such as electrical engineering and computer science to be successful. To help with this we created ready-to-use shape changing fabric samples for fashion designers. We explored this research gap through a preliminary user case study with seven experienced designers. Our results suggest design approaches for shape changing fabric samples that would assist non-technically skilled designers incorporating technology in their designs.
\end{abstract}

\section{CCS CONCEPTS}

- Human-centered computing $\rightarrow$ User interface toolkits.

\section{KEYWORDS}

interactive garments, fashion design, e-textile, smart textile, shapechanging interface, fabric, prototyping, toolkits

\section{ACM Reference Format:}

Daniela Ghanbari Vahid, Lee Jones, Audrey Girouard, and Lois Frankel. 2021. Shape Changing Fabric Samples for Interactive Fashion Design. In Fifteenth International Conference on Tangible, Embedded, and Embodied Interaction (TEI '21), February 14-17, 2021, Salzburg, Austria. ACM, New York, NY, USA, 7 pages. https://doi.org/10.1145/3430524.3440633

\section{INTRODUCTION}

Designing interactive garments presents unique challenges to researchers and designers because collaboration in this field is still relatively new and not part of traditional fashion design training [17]. Fashion designers and engineers have skills and knowledge that could complement each other during e-textile collaborations

Permission to make digital or hard copies of all or part of this work for personal or classroom use is granted without fee provided that copies are not made or distributed for profit or commercial advantage and that copies bear this notice and the full citation on the first page. Copyrights for components of this work owned by others than the author(s) must be honored. Abstracting with credit is permitted. To copy otherwise, or republish, to post on servers or to redistribute to lists, requires prior specific permission and/or a fee. Request permissions from permissions@acm.org.

TEI '21, February 14-17, 2021, Salzburg, Austria

(C) 2021 Copyright held by the owner/author(s). Publication rights licensed to ACM. ACM ISBN 978-1-4503-8213-7/21/02 ..\$15.00

https://doi.org/10.1145/3430524.3440633
[45], but with current tools, fashion designers often must already have engineering and computing knowledge to fully experiment and explore the material opportunities of interactive textiles. Designers without experience using actuated e-textiles or visualization media are limited in their ability to develop shape-changing wearable fashions [29]. In addition to considering factors such as fit, user comfort and aesthetics, interactive garment designers need to pay attention to other aspects of the design such as visibility of electronic elements and electronics reliability [36]. Based on these considerations, fashion designers typically collaborate with other professionals to implement technologies within their designs $[26,27,29]$. Yet, there can be communication barriers between researchers or developers; technology researchers need to understand designers' intentions to solve their problems efficiently [36].

We propose simplifying the process with ready-to-use interactive garment samples that could minimize the need for technical assistance in the design of shape-changing clothing. Previous work has explored how fashion designers would like to use colour-changing samples [9], but shape-changing samples are an underexplored area. In this work, we focus on inflatable shape-changing fabric samples with kinetic properties. We asked, "How can inflatable fabric samples help designers explore potential applications of shape-changing technology?" Our three samples are based on origami patterns that change their shape by folding and unfolding through the use of air pressure. We provided these fabric samples to seven designers without a technical background to identify possible advantages and disadvantages. Based on our qualitative findings of this first evaluation, we suggest design approaches for shape changing fabric samples, which could assist designers in independently using electronics in garment design.

\section{RELATED WORK}

Electronic textiles (e-textiles) are fabrics that incorporate electronic elements such as sensors and actuators to make textiles interactive [39]. One recent area of exploration, shape-changing actuation, provides several opportunities for fashion designers, although technical support for fabric actuation is not well documented.

\subsection{Shape-Changing Textile Actuation}

Availability of shape- and texture-changing devices is increasing [40]. In the field of fashion, this may lead to garments that: change shape based on the wearer's needs [11,38, 43], fit the body perfectly[28], 
create second skins [2], or display emotions and sensory aspects [7, 35].

Some approaches to developing self-folding mechanisms include using Nitinol wire and shape memory [2, 10, 44], pneumatically actuated soft composite material $[15,46]$ or heat-sealing inflatable [34]. The inflatable fabric option is becoming popular in the field of material design, where a shape-changing surface can be created by folding and unfolding or origami patterns with air pressure [33].

\subsection{Scaffolding Learning and Exploration}

Designing wearable garments often requires interdisciplinary collaborations [29, 36]. As noted earlier, considerations include fabric texture, garment fit, comfort and aesthetics, visibility of the elements, size, and reliability of electronics [26, 27]. However, fashion designers often lack electronics and programming backgrounds [36].

One approach to overcome this challenge is the use of toolkits to help collaborators develop functional prototypes. LittleBits[1], Craftec [18], and MakerWear [24] are toolkits that use magnetic or snappable components. LilyPad $[4,6]$ is a toolkit with sewable components that can be attached to textiles and better support sewing practices. The current limitation of the toolkit approach is that the often-hard components do not emulate how the envisioned garment might look or feel.

Another approach taken from the textile industry is the use of swatches or samples to demonstrate what e-textiles can do. This is best demonstrated in the use of swatchbooks such as those developed during the yearly e-textile swatch exchange [16], or the textile interface swatchbook [13] which is an interactive book of samples that can be plugged into a computer for demonstrations. The use of samples is particularly powerful for enabling designers to see what is possible and gather inspiration for their own work [47]. Wearable Bits [22] and Swatch-Bits [20]are examples of using swatch samples as a toolkit for prototyping ideas, but they lack shape changing actuation samples. Other researchers have used probes such as the colour-changing fabric prototype Ebb [9], and the Kino robotic dress prototype [23].

Some researchers have incorporated the use of mannequins to emulate fashion design practices. One example is Mannequette [42], an interactive mannequin with knobs to emulate digital and analog sensors but currently limited to light actuation. Wooden mannequins have been used for sketching and hand fabricating non-functional prototypes with the benefit that participants do not need to know how to build interactive prototypes to express their concepts [3].

There are several shape-changing toolkits for scaffolding learning for beginners, but these are not geared towards fabric samples and fashion design uses cases. Inflatibits [25] is a building blocks toolkit of inflatable modules for education. Pneuduino [33] is an Arduino-based programmable electromechanical toolkit that helps designers by allowing them to create such shape-changing structures without going through all the steps that are usually required for this kind of task.

In this case study, we explore how fashion designers without computing or engineering experience would use interactive shape changing swatches or samples with wooden mannequins. Our ultimate goal is to provide fashion designers tools that help them explore the technology without requiring the support of an engineering expert.

\section{THE PROTOTYPES}

We designed three shape-changing fabric prototypes based on origami art patterns that change their shape as they fold and unfold. Origami is a traditional Japanese art that transforms a flat sheet of paper into a 3D structure [37]. We selected origami patterns because they have a demonstrated and efficient structure for shape-changing movements through folding and unfolding [37], and provide fashionable aesthetic qualities. We applied three types of tessellations: the Eight-Crease Waterbomb, measuring 3.5 by 3.5 inches, which looks like blooming flowers that expand (Figure 1, top), the Six-Crease Waterbomb, measuring 3.5 by 4.5 inches, which changes from a flat piece to a ball (Figure 1, middle), and the Pyramid, measuring 5.5 by 5.5 inches, with a self-explanatory name (Figure 1, bottom) $[8,32]$. To set the origami folds in the fabric, we
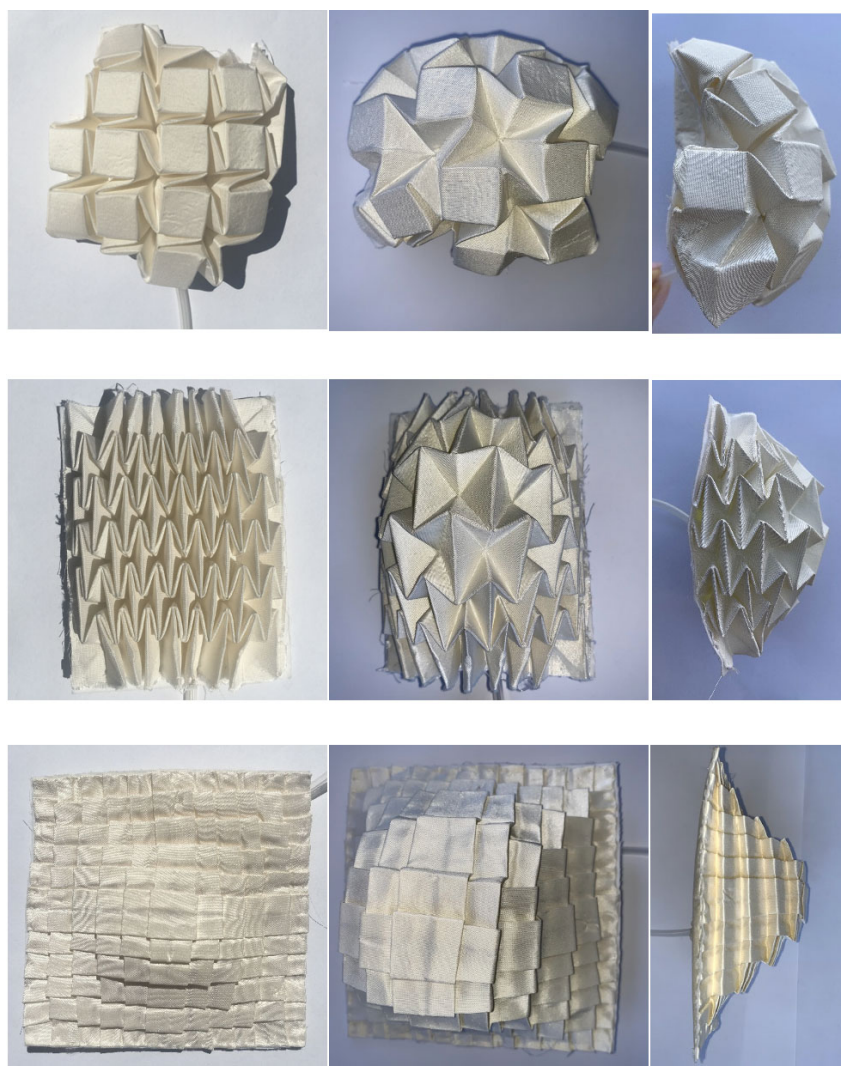

Figure 1: Eight-Crease Waterbomb (Top row); Six-Crease Waterbomb (Middle row); Pyramid (bottom row); non-inflated, view from the top (left column), inflated view from the top (middle column) and the side (right column).

drew the origami pattern on fusible web material and cut it into pieces. We ironed the pieces to the back of the fabric then sandwiched the fabrics between plastic origami sheets (mold, Figure 2, 
left) and secured the folds with wood clips (Figure 2, right). The fabric set for 2 or 3 days, and when we opened the clips, the fabric held the planned shape. We chose Silk Shantung woven fabric for

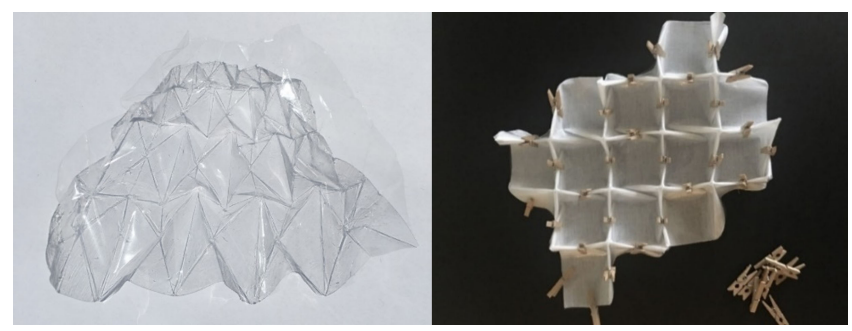

Figure 2: Building the shape-changing swatches (left) the mold, (right) securing the folds with wooden clips

the prototypes as it holds creases well and layered the fabric with fusible web material cut to the origami pattern. Each sample had a simple on/off switch to avoid the need for programming knowledge. The switch circuit included a small, handheld air compressor, a balloon and tubes to blow the air under the fabric to make the unfolding kinetic motion possible (Figure 3). The Six-Crease and Eight-Crease Waterbomb took about five seconds to inflate while the Pyramid required ten seconds. The Eight-Crease Waterbomb has a structure that enables self-unfolding with air pressure and it also looks like blooming flowers that expand. The Six-Crease Waterbomb changes shape from a flat piece to a ball and the shape is also known as the magic ball. All three samples took about 10 seconds to deflate.
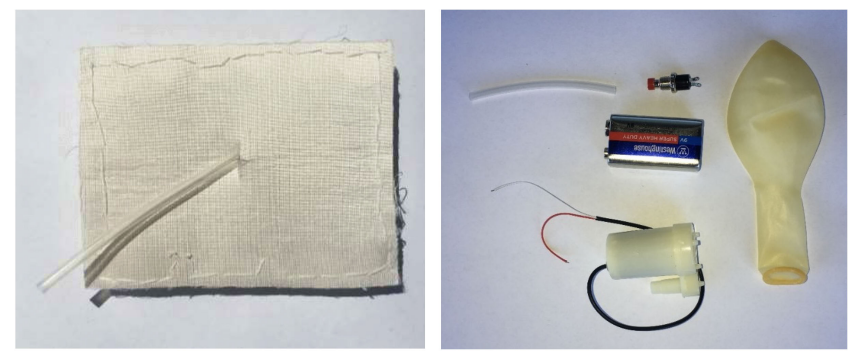

Figure 3: The back of the fabric with tube (left); the components used to implement the shape-changing movements (right).

\section{METHOD}

To determine how our shape changing fabric samples could help designers imagine and prototype future designs, we introduced participants to our ready-to-use, shape-changing fabrics. Our research methodology was reviewed and cleared by our institutional research ethics board. We conducted individual in-person design sessions, where we asked participants to interact with the fabric samples to design concepts for a garment or for any other kind of interactive piece. Each design activity lasted an average of thirty minutes. We demonstrated three shape-changing fabric samples to the participants and asked them to generate design concepts using our supplies [3]. We provided one yard of fabric, a 12-inch-tall mini mannequin, pins and scissors (Figure 4). We observed their design activities and asked questions about their interactions throughout the design session

\subsection{Participants}

We recruited seven participants for this study including fashion designers from Toronto Fashion Week as well as industrial designers who work with fabrics. Three participants had a college degree in fashion design, the other four were currently enrolled in or had a graduate degree. Five participants had less than 10 years of experience, one had 12 and one had more than 45 . All had experience in fabric design, four were fashion designers with experience in custom-made dresses, two had experience in sewing and tailoring and one had experience sketching garments and working with fabrics in industrial design. However, none had experience with merging technology and fashion, for example one participant noted, "I never interacted with the technology and I just work with traditional fabrics and cut them. But I believe that technology is going to make its way to fashion as well."

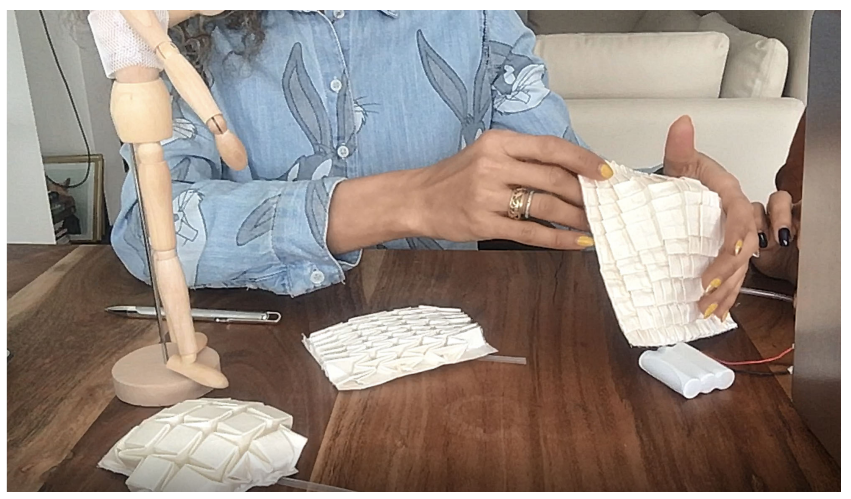

Figure 4: Study setup: a mannequin and three prototypes.

\subsection{Analysis}

We video recorded the study and transcribed the discussion manually. We used a descriptive coding method to analyze the study transcript [41] and identified general topic categories discussed in each session. We also noted observations from participants' interactions with the prototypes during each session. Our analysis included ethnographic thick descriptions of their responses, in line with Clifford Geertz's qualitative approach to including interpretive cultural contexts and meanings that provide insight into the factual accounts [12].

\section{RESULTS}

\subsection{Understanding the Samples}

We observed that all the participants seemed interested in seeing how the prototypes worked. P3 stated "I always think that we don't have enough of the combination of technology and fashion. There's not enough of it." Participants asked many questions about the technology involved in making them. P1 asked questions about 
cutting, sewing and the mechanics inside the fabric (Figure 5, left). Specifically, she wanted to know if she could cut the fabric in the shape that she wanted and if she could sew pieces together. She expected to have an instruction manual that explains how to cut and use the fabrics.

Participants interacted with each shape changing fabric prototype and evaluated its flexibility and softness (Figure 4). Most designed on the mini-mannequin (e.g. in Figure 5, right), but P1 and $\mathrm{P} 2$ put the fabric on their body. P1 placed the sample on her shoulders to see if it would look good as a jacket lapel.
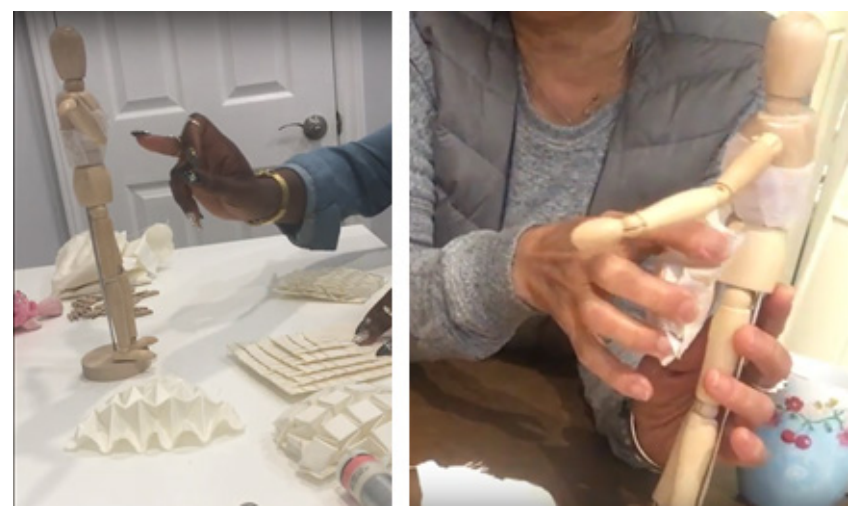

Figure 5: P1 wanted to stitch around the fabric and cut it as a corset (left);P2 expected that Eight-Crease Waterbomb fabric changed the length (right).

\subsection{Emotional and Functional Engagement}

Through analysis of our observations and the participants' comments and behaviours, we categorized that they perceived the prototypes as enabling Emotionally-Oriented and Functionally-Oriented engagement. These are not dichotomous categories, but more a categorization of the core perception of the opportunity.

For participant perceptions focused on the Emotionally-Oriented opportunities, the emotional and social aspects of using the fabrics were important to their design prospects. They mentioned the potential to use them on the red carpet or in a big event or a fashion show; they seemed to be interested in how the fabrics would impress people: "Six-Crease waterbomb is interesting and looks futuristic." All three responded that they were likely to recommend this prototype to another designer and would consider using them in their own design. One participant reacted negatively to the aesthetics of the fabric samples: P4 mentioned that, since these fabrics are blowing up to change shape, there is no aesthetically pleasing aspect to use: "I am not quite sure where you would go with this blowing up." Instead she focused on the functional aspect of the prototypes.

For participant perceptions focused on the Functionally-Oriented opportunities, the functional applications of using the shape-changing fabrics were important to their design prospects. They identified the ability to protect the wearer from falling or from bad weather conditions. P4 focused on using the fabrics for protective purposes, "If something bubbles up and it supports the body that would be a pretty smart way to use these fabrics. This technology can protect the wearer from a dangerous act or a stunt and having a bad fall." P3 wanted to use it for an outerwear garment to keep the body warm when inflated due to the air in between the garment and the body. This group was interested in working on traditional garments and fabric in more conventional ways. Furthermore, one of the main functional concerns of these participants was that the garments could not be used for everyday outerwear because of the lack of social acceptability.

Participants also proposed using the samples for non-body purposes such as furniture. P4 said, "It also can serve a purpose outside of the body like using it on the couch, it would be really cool as an interactive canopy or for your patio in the backyard. On rainy days have these shape changing fabrics on top for better protection. When your nice little gazebo has this fabric out when it starts raining, the fabric changes their shape and they cover the roof. We can probably do a lot with these fabrics!"

Functionally-oriented opportunities also included safety practicalities. One participant mentioned that she was worried about the safety of the wearer and asked questions: "Is this detachable? The customers are not going to electrocute themselves?! Is there going to be a risk? How about if it is raining?"

\subsection{Foldable Structure}

All seven participants exhibited positive attention at the beginning of the study: they showed interest in how the prototype worked and asked questions about the technology involved in making the prototypes. Participants held and folded each kinetic fabric prototype; they touched the fabrics to evaluate their flexibility and the softness of the material (Figure 6).
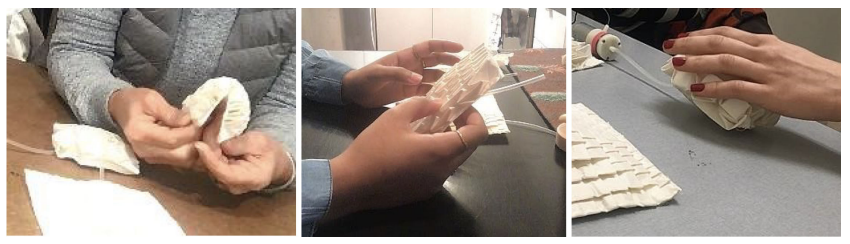

Figure 6: Participants tangibly explored the features and affordances of the swatches

Shape-changing seemed to be a pleasing feature to participants, even if some noted the limited flexibility of our samples. Pattern preference was equally distributed among participants; three liked the Six-Crease Waterbomb due to its flexibility for ease of use; two preferred the Eight-Crease Waterbomb due to its appearance, and three selected the Pyramid due to its limited movement and more obvious shape change, producing a surprise effect.

Participants noted that it would be suitable for the bottom parts of the body rather than the top. They explained that they would prefer not to use the prototypes on certain parts of the body-specifically arms, waistline and stomach-because it could limit movement abilities, make the wearers look big and also decrease comfort. For this reason, participants ended up designing for a similar body part across the different design sessions. Three participants suggested using these fabrics on the shoulders: P3 chose the Pyramid for the 

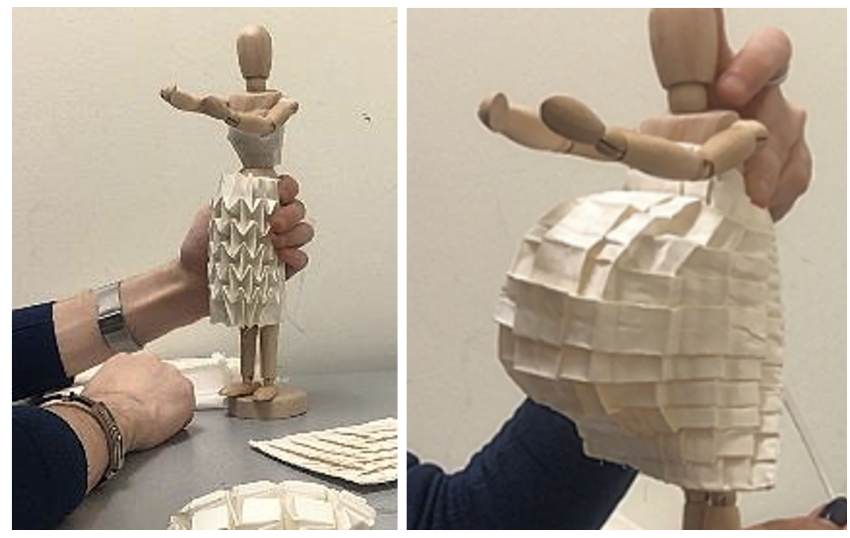

Figure 7: Many participants made a skirt with the Six-Crease Waterbomb shape-changing fabric (left); P7 made a pregnant lady using the Pyramid fabric (right).

shoulders for "a female Gladiator style”. P3 also designed a day and evening wear skirt that would be flat during the day and could puff up during the evening. P7 said he would use the Six-Crease and Eight-Crease Waterbomb fabrics on the bottom of the dress rather than on the top, and not close to the arms. He also said that the Pyramid fabric is suitable for the top. He worked on a pregnant lady on the mini mannequin, and stated, "It's kind of cute. I mean it's not bad. It's interesting, like a piece of art." (Figure 7, right).

Five participants made a skirt on the mini mannequin with the Six-Crease Waterbomb sample (Figure 7, left), often creating a puffy skirt. Five participants suggested making a push-up bra due to the light weight and size changing properties. Three participants mentioned that the garments would not be suitable for off-the-rack purposes. One stated, "I would use this material for ready-to-wear clothes for different events." Another designed a wedding dress to overcome current issues with weight and size, and stated, "If there is air when you wear it and then the air goes off and then you can store it."

\subsection{Creativity}

In the short time they worked with the samples, they generated concepts that extend the possibilities for everyday fashion designs. Two participants introduced the use of the fabrics for artistic-oriented designs such as the pregnant lady and gladiator lady garment. Two wanted to design a dress to surprise audiences at an event or fashion show. P7 imagined the audience response, "The dress is becoming bigger and bigger in the public is like, wow!” This is in line with findings regarding a colour changing wearable provoking intrigue [9].

\section{DISCUSSION}

Tangible exploration is an integral part of how fashion designers evaluate and ideate on materials and potential applications. As researcher Pauline van Dogen describes, fashion involves "material processes and embodied practices" [43]. In our project, we found that tangible exploration of shape-changing swatches provided several insights into both approaches that could lead to recommendations for other researchers working on interactive fashion toolkits.

\subsection{Opportunities with Shape Change}

There are many toolkits for wearable prototyping and co-design, but none currently support or include shape changing elements. As noted, our participants seemed to enjoy exploring shape changing concepts, suggesting that shape-changing samples could be an interesting feature to add to wearable e-textile fashion prototyping kits. Participants also emphasized the importance of the fabric samples feeling like fabric, and the most repeated complaint was sample rigidity. This suggests that the component rigidity of many of the available e-textile and wearable toolkits would not be suitable for fashion collaborations and that QuiltSnaps [5] or e-textile swatchbooks [16] could be more suitable for this context.

It is also critical for toolkits to consider the process of fashion designers, which often work with fabric bolts that can be cut. This is quite different from circuits, which typically cannot be cut freely and maintain their integrity. This need to modify of the samples, as brought up by $\mathrm{P} 1$, is of particular importance. Overall, future researchers exploring shape-change in fashion should ensure that their samples are soft, feel like fabric and can be modified as part of working with the samples.

\subsection{The Importance of Scale in Fashion Design}

Using a mini-mannequin provided a framework for participants to imagine different scenarios while prototyping. In particular, it helped them to imagine large scale interventions such as a skirt, even with a small sample, but also limited the types of interactions due to the scale of the model. In contrast, in fashion design the physical prototyping process of toile involves using a cheaper fabric such as canvas to mock-up a design on a life-scale mannequin [30]. Having mannequins and samples at different scales could help to better support the processes and embodied practices our participants were trying to explore, for example by placing a swatch on their own body to explore their design concept (e.g.[42]. Designers mentioned this preference to designing on a human-sized mannequin and also would have wanted the opportunity to cut the fabrics into different shapes. They said that working with small samples on a small mannequin was a limitation. Interestingly, only one of them was concerned about the scale of the fabrics and she stated, "I don't like to have the samples in larger of the scale but if you keep the scale small and give me a large piece of fabric then I am happy." Another approach would be to develop different sized swatches such as Wearable Bits [22], that are integrated as part of a larger design ecosystem.

Also, though swatches are common exploration tools in fashion design, many fashion designers are used to working with fabrics in a cut-and-sew manner which is not compatible with many interactive swatches (such as our inflatables). For future toolkits, considering how swatch examples could be cut and sewn, and designing them so they can be cut without damaging the circuits, would likely enable fashion designers to more easily envision how they could incorporate these technologies into their work. 


\subsection{Design Activities for Different Stages and Purposes}

The samples were useful for demonstrating the possibilities of shape-changing fabrics and for quickly building prototypes to express design ideas. We learned that they can be approached as tools that could affect the emotional and social impressions of designing with and for shape-changing clothing. They could also be used as instruments for enabling functional applications for increasing the usefulness of protective outerwear and expandable furniture.

In contrast to our initial expectations, the concepts were conservative and mostly around a narrow design space. This suggests that the samples alone may not be sufficient for shape-changing ideation, and that participants might benefit from more time to reflect on samples and additional related design activities. To do so the shape-changing samples could be combined with activities such as moodboards [19], card sorting [31], ideation decks [14], and sketching [21, 47] to help participants become immersed in new contexts.

\subsection{Limitations}

We had a very small sample of designers, due to their limited availability and time constraints. In addition, the designers who participated only had a short time to interact with the fabric samples as we could not produce enough samples to let them become familiar with them prior to the design sessions. We consider this a preliminary study and propose developing a series of kits that could be distributed ahead of time to a broader sample population for future studies.

\section{CONCLUSION}

This preliminary case study explores how shape-changing inflatable fabric samples can help fashion designers explore affordances and opportunities for designing with shape-changing samples without engineering or computing assistance. We explored this research gap through a preliminary user case study with seven experienced designers. We created three origami patterns that fold and unfold and observed designers interacting with these samples in a design session. We found that designers demonstrated both emotionallyoriented and functionally-oriented approaches to the potential for the samples to help them envision shape-changing clothing and furniture design concepts. Our results suggest addressing these design perspectives in developing shape-changing fabric samples that could assist fashion designers with little to no experience with electronics incorporating technology in their designs.

We propose that samples of shape-changing fabrics, similar to our prototypes, could be a potential solution to help designers integrate technology into their current processes and practices, without the need for external help. Specifically, future work should include the creation of a shape-changing fabric sample kit, which provides larger and more varied patterns of interactive sample materials and instructions for using the samples that show examples of applications and illustrate steps for ideating with the prototypes provided. These kits could be on loan to enable designers to experiment with them. This may inspire more varied interactive garments.

\section{ACKNOWLEDGMENTS}

This work was supported and funded by the National Sciences and Engineering Research Council of Canada (NSERC) through the Collaborative Learning in Usability Experiences Create grant (2015-465639).

\section{REFERENCES}

[1] Ayah Bdeir. 2009. Electronics as material: littleBits. In Proceedings of the 3rd International Conference on Tangible and Embedded Interaction. 397-400. https: //doi.org/10.1145/1517664.1517743

[2] Joanna Berzowska and Marcelo Coelho. 2005. Kukkia and vilkas: Kinetic electronic garments. In Ninth IEEE International Symposium on Wearable Computers (ISWC'05). IEEE, 82-85. https://doi.org/10.1109/ISWC.2005.29

[3] Adrien Bousseau, Theophanis Tsandilas, Lora Oehlberg, and Wendy E Mackay. 2016. How novices sketch and prototype hand-fabricated objects. In Proceedings of the 2016 CHI Conference on Human Factors in Computing Systems. 397-408. https://doi.org/10.1145/2858036.2858159

[4] Leah Buechley, Mike Eisenberg, Jaime Catchen, and Ali Crockett. 2008. The LilyPad Arduino: using computational textiles to investigate engagement, aesthetics, and diversity in computer science education. In Proceedings of the SIGCHI conference on Human factors in computing systems. 423-432. https: //doi.org/10.1145/1357054.1357123

[5] Leah Buechley, Nwanua Elumeze, Camille Dodson, and Michael Eisenberg. 2005. Quilt snaps: A fabric based computational construction kit. In IEEE International Workshop on Wireless and Mobile Technologies in Education (WMTE'05). IEEE, 3-pp. https://doi.org/10.1109/WMTE.2005.55

[6] Leah Buechley and Benjamin Mako Hill. 2010. LilyPad in the wild: how hardware's long tail is supporting new engineering and design communities. In Proceedings of the 8th ACM conference on designing interactive systems. 199-207. https: //doi.org/10.1145/1858171.1858206

[7] Stephen Cass. 2016. Anouk Wipprecht: dynamic dresses merge high fashion and technology [Resources]. IEEE Spectrum 53, 2 (2016), 19-20.

[8] Yan Chen, Huijuan Feng, Jiayao Ma, Rui Peng, and Zhong You. 2016. Symmetric waterbomb origami. Proceedings of the Royal Society A: Mathematical, Physical and Engineering Sciences 472, 2190 (2016), 20150846.

[9] Laura Devendorf, Joanne Lo, Noura Howell, Jung Lin Lee, Nan-Wei Gong, M Emre Karagozler, Shiho Fukuhara, Ivan Poupyrev, Eric Paulos, and Kimiko Ryokai. 2016. " I don't Want to Wear a Screen" Probing Perceptions of and Possibilities for Dynamic Displays on Clothing. In Proceedings of the 2016 CHI Conference on Human Factors in Computing Systems. 6028-6039. https://doi.org/10.1145/ 2858036.2858192

[10] Christine Dierk, Sarah Sterman, Molly Jane Pearce Nicholas, and Eric Paulos. 2018. Häiriö: Human hair as interactive material. In Proceedings of the Twelfth International Conference on Tangible, Embedded, and Embodied Interaction. 148157. https://doi.org/10.1145/3173225.3173232

[11] Julia C Duvall, Lucy E Dunne, Nicholas Schleif, and Brad Holschuh. 2016. Active" hugging" vest for deep touch pressure therapy. In Proceedings of the 2016 ACM international joint conference on pervasive and ubiquitous computing: adjunct. 458-463. https://doi.org/10.1145/2968219.2971344

[12] Clifford Geertz. 1973. The interpretation of cultures. Vol. 5019. Basic books.

[13] Scott Gilliland, Nicholas Komor, Thad Starner, and Clint Zeagler. 2010. The textile interface swatchbook: Creating graphical user interface-like widgets with conductive embroidery. In International Symposium on Wearable Computers (ISWC) 2010. IEEE, 1-8. https://doi.org/10.1109/ISWC.2010.5665876

[14] Michael Golembewski and Mark Selby. 2010. Ideation decks: a card-based design ideation tool. In Proceedings of the 8th ACM Conference on Designing Interactive Systems. 89-92. https://doi.org/10.1145/1858171.1858189

[15] Liang He, Cheng Xu, Ding Xu, and Ryan Brill. 2015. PneuHaptic: delivering haptic cues with a pneumatic armband. In Proceedings of the 2015 ACM International Symposium on Wearable Computers. 47-48. https://doi.org/10.1145/2802083. 2802091

[16] Anja Hertenberger, Barbro Scholz, Beam Contrechoc, Becky Stewart, Ebru Kurbak, Hannah Perner-Wilson, Irene Posch, Isabel Cabral, Jie Qi, Katharina Childs, Kristi Kuusk, Lynsey Calder, Marina Toeters, Marta Kisand, Martijn ten Bhömer, Maurin Donneaud, Meg Grant, Melissa Coleman, Mika Satomi, Mili Tharakan, Pauline Vierne, Sara Robertson, Sarah Taylor, and Troy Robert Nachtigall. 2014. 2013 E-Textile Swatchbook Exchange: The Importance of Sharing Physical Work. In Proceedings of the 2014 ACM International Symposium on Wearable Computers: Adjunct Program (Seattle, Washington) (ISWC '14 Adjunct). Association for Computing Machinery, New York, NY, USA, 77-81. https://doi.org/10.1145/2641248.2641276

[17] Nancy J Hodges and Albert N Link. 2019. Innovation by design. Small Business Economics 52, 2 (2019), 395-403. 
[18] Ben Jelen, Anne Freeman, Mina Narayanan, Kate M Sanders, James Clawson, and Katie A Siek. 2019. Craftec: Engaging older adults in making through a craftbased toolkit system. In Proceedings of the Thirteenth International Conference on Tangible, Embedded, and Embodied Interaction. 577-587. https://doi.org/10.1145/ 3294109.3295636

[19] Lee Jones, Meghrik Isagholi, Elizabeth Meiklejohn, Snow Xu, Kara Truskolawski, Jessica Hayon, Grace Jun, Pinar Guvenc, and Christina Mallon-Michalove. 2020 Hack-Ability: Using Co-Design to Develop an Accessible Toolkit for Adding Pockets to Garments. In Proceedings of the 16th Participatory Design Conference 2020-Participation (s) Otherwise-Volume 2. 95-99. https://doi.org/10.1145/3384772. 3385124

[20] Lee Jones, Sara Nabil, and Audrey Girouard. 2020. Swatch-bits: Prototyping E-textiles with Modular Swatches. In Proceedings of the Fourteenth International Conference on Tangible, Embedded, and Embodied Interaction. 893-897. https: //doi.org/10.1145/3374920.3374971

[21] Lee Jones, Sara Nabil, and Audrey Girouard. 2021. Wearable Crazy Eights: Wearable Ideation Methods for Encouraging Divergent Design Concepts. In Fifteenth International Conference on Tangible, Embedded, and Embodied Interaction (TE '21), February 14-17, 2021, Salzburg, Austria. Association for Computing Machinery, New York, NY, USA. https://doi.org/10.1145/3430524.3442464

[22] Lee Jones, Sara Nabil, Amanda McLeod, and Audrey Girouard. 2020. Wearable Bits: scaffolding creativity with a prototyping toolkit for wearable e-textiles. In Proceedings of the Fourteenth International Conference on Tangible, Embedded, and Embodied Interaction. 165-177. https://doi.org/10.1145/3374920.3374954

[23] Hsin-Liu Kao, Deborah Ajilo, Oksana Anilionyte, Artem Dementyev, Inrak Choi, Sean Follmer, and Chris Schmandt. 2017. Exploring interactions and perceptions of kinetic wearables. In Proceedings of the 2017 Conference on Designing Interactive Systems. 391-396. https://doi.org/10.1145/3064663.3064686

[24] Majeed Kazemitabaar, Jason McPeak, Alexander Jiao, Liang He, Thomas Outing, and Jon E Froehlich. 2017. Makerwear: A tangible approach to interactive wearable creation for children. In Proceedings of the 2017 chi conference on human factors in computing systems. 133-145. https://doi.org/10.1145/3025453.3025887

[25] Christopher Kopic and Kristian Gohlke. 2016. InflatiBits: A Modular Soft Robotic Construction Kit for Children. In Proceedings of the TEI'16: Tenth International Conference on Tangible, Embedded, and Embodied Interaction. 723-728. https: //doi.org/10.1145/2839462.2872962

[26] L Ashok Kumar and C Vigneswaran. 2015. Electronics in textiles and clothing: design, products and applications. CRC Press.

[27] Yuhua Li, Jianwei Zhang, Ming Chen, Haopeng Lei, Guoliang Luo, and Yan Huang. 2019. Shape based local affine invariant texture characteristics for fabric image retrieval. Multimedia Tools and Applications 78, 11 (2019), 15433-15453. https://doi.org/10.1007/s11042-018-6936-y

[28] Jason Lin, Jasmine Zhou, and Helen Koo. 2015. Enfold: clothing for people with cerebral palsy. In Adjunct Proceedings of the 2015 ACM International foint Conference on Pervasive and Ubiquitous Computing and Proceedings of the 2015 ACM International Symposium on Wearable Computers. 563-566. https://doi.org/ $10.1145 / 2639108.2639123$

[29] Jane McCann and David Bryson. 2009. Smart clothes and wearable technology. Elsevier.

[30] Holly McQuillan, Timo Rissanen, and Julian Roberts. 2013. The cutting circle: how making challenges design. Research fournal of Textile and Apparel 17, 1 (2013), 39. https://doi.org/10.1108/RJTA-17-01-2013-B004

[31] Sara Nevay and Christopher SC Lim. 2015. The Role of Co-Design in Wearables Adoption. In Contemporary Ergonomics and Human Factors 2015: Proceedings of the International Conference on Ergonomics \& Human Factors 2015, Daventry, Northamptonshire, UK, 13-16 April 2015, Vol. 109.

[32] Non numerical Processing Algorithms Laboratory. 2014. A Design System for Orthogonal Pleat Tessellation. http://www.npal.cs.tsukuba.ac.jp/projects/2014/ pleat tessellation/index.html. accessed: 2019-04-01.

[33] Jifei Ou, Felix Heibeck, and Hiroshi Ishii. 2016. TEI 2016 Studio: Inflated Curiosity. In Proceedings of the TEI'16: Tenth International Conference on Tangible, Embedded and Embodied Interaction. 766-769. https://doi.org/10.1145/2839462.2854119

[34] Jifei Ou, Mélina Skouras, Nikolaos Vlavianos, Felix Heibeck, Chin-Yi Cheng, Jannik Peters, and Hiroshi Ishii. 2016. aeroMorph-heat-sealing inflatable shapechange materials for interaction design. In Proceedings of the 29th Annual Symposium on User Interface Software and Technology. 121-132. https://doi.org/10. 1145/2984511.2984520

[35] Birce Ozkan. 2015. Fall: an interactive garment that mimics nature's responsive system of defoliation through an embedded electronic mechanism. In Adjunct Proceedings of the 2015 ACM International foint Conference on Pervasive and Ubiquitous Computing and Proceedings of the 2015 ACM International Symposium on Wearable Computers. 617-621. https://doi.org/10.1145/2800835.2801675

[36] Rebeccah Pailes-Friedman. 2016. Smart textiles for designers: Inventing the future of fabrics. Laurence King Publishing London.

[37] Edwin A Peraza-Hernandez, Darren J Hartl, Richard J Malak Jr, and Dimitris C Lagoudas. 2014. Origami-inspired active structures: a synthesis and review. Smart Materials and Structures 23, 9 (2014), 094001.
[38] Laura Perovich, Philippa Mothersill, and Jennifer Broutin Farah. 2014. Awakened apparel: embedded soft actuators for expressive fashion and functional garments. In Proceedings of the 8th International Conference on Tangible, Embedded and Embodied Interaction. 77-80. https://doi.org/10.1145/2540930.2540958

[39] Irene Posch, Liza Stark, and Geraldine Fitzpatrick. 2019. eTextiles: reviewing a practice through its tool/kits. In Proceedings of the 23rd International Symposium on Wearable Computers. 195-205. https://doi.org/10.1145/3341163.3347738

[40] Anne Roudaut, Abhijit Karnik, Markus Löchtefeld, and Sriram Subramanian. 2013. Morphees: toward high" shape resolution" in self-actuated flexible mobile devices. In Proceedings of the SIGCHI Conference on Human Factors in Computing Systems. 593-602. https://doi.org/10.1145/2470654.2470738

[41] Johnny Saldaña. 2015. The coding manual for qualitative researchers. Sage.

[42] Teddy Seyed and Anthony Tang. 2019. Mannequette: Understanding and Enabling Collaboration and Creativity on Avant-Garde Fashion-Tech Runways. In Proceedings of the 2019 on Designing Interactive Systems Conference. 317-329. https://doi.org/10.1145/3322276.3322305

[43] Pauline Louisette van Dongen. 2019. A designer's material-aesthetics reflections on fashion and technology. (2019).

[44] Luisa von Radziewsky, Antonio Krüger, and Markus Löchtefeld. 2015. Scarfy: augmenting human fashion behaviour with self-actuated clothes. In Proceedings of the Ninth International Conference on Tangible, Embedded, and Embodied Interaction. 313-316. https://doi.org/10.1145/2677199.2680568

[45] Susan M Watkins and Lucy Dunne. 2015. Functional clothing design: From sportswear to spacesuits. Bloomsbury Publishing USA.

[46] Lining Yao, Ryuma Niiyama, Jifei Ou, Sean Follmer, Clark Della Silva, and Hiroshi Ishii. 2013. PneUI: pneumatically actuated soft composite materials for shape changing interfaces. In Proceedings of the 26th annual ACM symposium on User interface software and Technology. 13-22. https://doi.org/10.1145/2501988.2502037

[47] Clint Zeagler, Stephen Audy, Scott Pobiner, Halley Profita, Scott Gilliland, and Thad Starner. 2013. The electronic textile interface workshop: Facilitating interdisciplinary collaboration. In 2013 IEEE International Symposium on Technology and Society (ISTAS): Social Implications of Wearable Computing and Augmediated Reality in Everyday Life. IEEE, 76-85. https://doi.org/10.1109/ISTAS.2013.6613105 